Postactivation potentiation

\title{
Postactivation potentiation: role in performance
}

\section{Sale}

\section{The role of postactivation potentiation in enhancement of strength and speed performance requires further research}

$\mathrm{P}$

ostactivation potentiation (PAP) also known as activity-dependent potentiation, is an increase in muscle isometric twitch and low frequency tetanic force following a "conditioning" activity. ${ }^{12}$ Examples of conditioning activity are a series of evoked isometric twitches (staircase or treppe), an evoked isometric tetanic contraction (post-tetanic potentiation), a sustained isometric maximal voluntary contraction (MVC), and a series of dynamic contractions. In fact, any type of contractile activity is likely to activate the mechanism of $\mathrm{PAP}$ - that is, phosphorylation of myosin regulatory light chains, which increases $\mathrm{Ca}^{2+}$ sensitivity of the myofilaments. ${ }^{2}$ The result is an amplified level of myosin cross bridge activity in response to submaximal concentrations of myoplasmic $\mathrm{Ca}^{2+}$. A notable feature of PAP is that it has no effect on the force of high frequency tetanic isometric contractions, because in such contractions a "saturating" concentration of $\mathrm{Ca}^{2+}$ is attained, making any increase in $\mathrm{Ca}^{2+}$ sensitivity inconsequential. Although less studied, PAP also increases the force of shortening (concentric) contractions, ${ }^{3}$ and the highest frequency at which PAP is effective is greater for rapid shortening (concentric) contractions than for isometric contractions. ${ }^{4}$

On the basis of the foregoing, it would appear that PAP has its greatest effect on performance in which motor units are firing at relatively low frequencies, such as endurance exercise. Endurance performance typically consists of submaximal contractions that are repeated for prolonged periods. From the beginning of performance, the contractions themselves would activate the mechanism responsible for PAP. In these submaximal contractions, motor units would be discharging at relatively low rates; thus, the force output of the motor units should be increased by PAP. If a constant force has to be maintained, motor unit firing rates would have to decrease to compensate for the increased force. A decrease in motor unit firing rate could, by reducing the number of nerve impulses and muscle action potentials per unit time, delay the onset of fatigue. For example, the risk of neuromuscular transmission failure, muscle action potential propagation failure, and excitation-contraction coupling impairment is increased in proportion to the frequency of nerve/ muscle action potentials that must be sustained. By reducing the required frequency, PAP should delay fatigue. In sustained exercise, there is also the possibility of impaired "central drive" to motoneurones. By increasing the force output for a given motor unit firing rate, PAP could relieve the burden of maintaining a high level of excitation of the motoneurones.

PAP may have a special role in compensating for the impaired excitation-contraction coupling that occurs with fatigue. Impaired excitation-contraction coupling is responsible for low frequency fatigue- that is, a disproportionate loss of low frequency tetanic force. This is the exact opposite of PAP, which is a disproportionate increase in low frequency tetanic force. Thus, PAP may compensate for low frequency fatigue. ${ }^{5}$ Although many endurance activities, such as running, cycling, swimming, consist of repeated brief concentric or eccentric-concentric actions in which motor units discharge briefly at fairly high rates, it should be recalled that in concentric (compared with isometric) actions, PAP and perhaps low frequency fatigue can act at higher frequencies.

Strength and speed performance typically requires that, in a brief maximal effort, all relevant motor units are recruited and firing at maximum possible rates. PAP would appear to offer little benefit when motor units are discharging at very high rates, because it cannot increase high frequency force. However, it has an additional effect; it can increase isometric rate of force development, even at relatively high stimulation frequencies at which isometric force is not increased by PAP. ${ }^{1}$ Furthermore, in fast shortening contractions, the effect of PAP is present at still higher frequencies. ${ }^{4}$ Whether PAP is effective at the motor unit firing rates attained in fast "ballistic" performance is at present not known. Nevertheless, by increasing the rate of force development, it could enhance performance in activities such as jumping, kicking, and throwing.

There are reports ${ }^{6-8}$ of improved performance of this nature after a conditioning activity such as isometric MVCs or a set of repetitions with a heavy weight, but the results have been inconsistent. The inconsistency may be the result of variation in the performance to be improved, the conditioning activity, and the time interval between the conditioning activity and the performance.

In exploiting PAP to enhance strength and speed performance, two dilemmas must be resolved. Firstly, a more intense and prolonged conditioning activity may activate the PAP mechanism to a greater extent, but it also produces greater fatigue. The second dilemma is that the longer the recovery period between the end of the conditioning activity and the beginning of performance, the greater the recovery from fatigue, but also the greater the decay of the PAP mechanism. The two dilemmas can only be resolved by trial and error. In one study $^{9}$ for example, the recovery period after a 10 second isometric MVC was only 15 seconds, so that performance (dynamic knee extension) would begin when PAP (assessed by the force of twitch contraction) was still near its maximum. However, performance was actually depressed because of fatigue induced by the conditioning activity. This was an example of low frequency (isometric twitch) force being increased at the same time as high frequency (concentric) force, and velocity was depressed. If a longer recovery period had been selected, say three minutes, performance may have been improved as suggested by some studies. ${ }^{67}$

An additional consideration is that, when the performance is a series of contractions, the contractions themselves have a cumulative effect in mobilising the PAP mechanism. ${ }^{9}{ }^{10}$ This may partly explain the progressive increase in performance observed in a series of jumps ${ }^{8}$ or dynamic knee extensions. ${ }^{9}{ }^{10}$ It has also been shown that the effects of a conditioning activity and repeated performance have an additive effect on the magnitude of PAP, at least over a few repetitions of the performance. ${ }^{9}$ In fact, if the performance consists of enough trials, the PAP induced by the trials themselves may rival that of the conditioning activity, making the latter unnecessary..$^{10}$ Again, all of this has to be sorted out by trial 
and error experiments, which should be the basis for future research.

Br J Sports Med 2004;38:386-387. doi: 10.1136/bjsm.2004.003392

Correspondence to: Dr Sale, Department of Kinesiology, McMaster University, Ivor Wynne Centre, Room AB 117, Hamilton, Ontario L8S 4L8, Canada; saled@mcmaster.ca

\section{REFERENCES}

1 Sale DG. Postactivation potentiation: role in human performance. Exerc Sport Sci Rev 2002;30:138-43.
2 Macintosh BR. Role of calcium sensitivity modulation in skeletal muscle performance. News Physiol Sci 2003; 18:222-5.

3 Macintosh BR, Bryan SN. Potentiation of shortening and velocity of shortening during repeated isotonic tetanic contractions in mammalian skeletal muscle. Pflugers Arch 2002;443:804-12.

4 Abbate F, Sargeant AJ, Verdijk PW, et al. A Effects of high-frequency initial pulses and posttetanic potentiation on power output of skeletal muscle. J Appl Physiol 2000;88:35-40.

5 Rassier DE. The effects of length on fatigue and twitch potentiation in human skeletal muscle. Clin Physiol 2000;20:474-82

6 Young WB, Jenner A, Griffiths K. Acute enhancement of power performance from heavy load squats. J Strength Cond Res 1998;12:82-4.
7 French DN, Kraemer WJ, Cooke CB. Changes in dynamic exercise performance following a sequence of preconditioning isometric muscle actions. J Strength Cond Res 2003:17:678-85

8 Güllich A, Schmidtbleicher D. Short-term potentiation of power performance induced by maximal voluntary contractions. XVth Congress of the International Society of Biomechanics 1995:348-9.

9 Gossen ER, Sale DG. Effect of postactivation potentiation on dynamic knee extension performance. Eur J Appl Physiol 2000;83:524-30.

10 Hughes SC, Gossen ER, Sale DG. Effect of postactivation potentiation on dynamic knee extension performance. Can J Appl Physiol 2001;26:486.

\section{ELECTRONIC PAGES}

\section{BJSM Online case reports: http://bjsm.bmijournals.com/}

7

he following electronic only articles are published in

conjunction with this issue of BJSM.

\section{Desbaric air embolism during diving - an unusual complication of Osler-Weber-Rendu disease Y-L Hsu, H-C Wang, P-C Yang}

Cerebral manifestations of Osler-Weber-Rendu disease (OWRD, hereditary haemorrhagic telangiectasia) including telangiectases, venous malformations, and arteriovenous malformations, are usually under-recognised. The highest complication rate is observed in high flow cerebral arteriovenous malformations, which may present with headache, epilepsy, ischaemia, or haemorrhage. Cerebral air embolism during self-contained underwater breathing apparatus (scuba) diving as the first manifestation of pulmonary arteriovenous malformation (PAVM) in OWRD patients has never been reported before. Here we report a 31 year old male who presented desbaric air embolism as the first manifestation of PAVM. As far as we know, this is the first such case published in English medical literature.

(Br J Sports Med 2004;38:e6) http://bjsm.bmjjournals.com/ cgi/content/full/38/4/e6

\section{Video illustration of staple gun use to rapidly repair on-field head laceration J W Orchard}

A professional rugby league player sustained a left eyebrow laceration during a match which immediately started to bleed. Within seconds he was taken to the sideline and had the laceration closed with six staples by the team doctor. Bleeding was arrested and he returned to play, taking the ball within $80 \mathrm{~s}$ of suffering the initial laceration, and within $40 \mathrm{~s}$ of the stapling procedure. This sequence was captured on video and appeared on television. The staples were removed after the match and the wound sutured. Repair of the wound was uneventful. The staple gun allows bleeding lacerations to be closed within seconds and for players to safely and quickly return to play, whilst minimising the risk of blood-borne infection transmission.

(Br J Sports Med 2004;38:e7) http://bjsm.bmjjournals.com/ cgi/content/full/38/4/e7

\section{Subdural haematoma associated with an arachnoid cyst after repetitive minor heading injury in ball games}

A K Demetriades, A W McEvoy, N D Kitchen

We report the case of a chronic subdural haematoma caused by repetitive heading of a football which led to the diagnosis of a middle fossa arachnoid cyst. The association between arachnoid cysts and subdural haematoma is discussed as are safety implications in sporting injuries.

(Br J Sports Med 2004;38:e8) http://bjsm.bmjjournals.com/ cgi/content/full/38/4/e8 